\title{
O futuro do sistema de saúde na espanha: será possível financiá-lo sem reformas estruturais?
}

\section{The future of the health system in spain:will it be possible to finance it without structural reforms?}

\section{El futuro del sistema sanitario en españa: será posible financiarlo sin reformas estructurales?}

Joan Barrubés ${ }^{1}$

Manel Peiró ${ }^{2}$

\begin{abstract}
RESUMO: Os sistemas de saúde contribuíram claramente para melhorar o estado de saúde da população, mas a taxa de crescimento dos gastos em saúde, superior à do crescimento do PIB nos países desenvolvidos, levanta questões sobre sua sustentabilidade. No entanto, a análise da sustentabilidade tende a ser parcial: concentra-se nos gastos com a saúde e, raramente, na cobertura e nas receitas fiscais do sistema de saúde, um ponto crítico no caso da Espanha. Neste artigo analisamos o impacto da crise econômica na sustentabilidade do sistema de saúde espanhol, e não apenas seu impacto econômico, mas também em termos de aumento de saúde e desigualdades. E, finalmente, uma estimativa do crescimento dos gastos de saúde pública em Espanha para os próximos anos em um cenário de reforma e um cenário de crescimento sem mudanças estruturais, para trazer para fora o custo de não fazer nada é feito. Mudanças e reformas tornam-se imperativas, mas isso requer um clima de discussão calma e aberta que envolva todos os atores do processo, abordando os problemas reais.
\end{abstract}

Palavras chaves: Gastos com saúde. Financiamento. crise econômica.

1 Sociodirector de Antares Consulting. Profesorasociadodel Departamento de Dirección de Personas y Organización de ESADE, España. Email: jbarrubes@antares-consulting.com

2 Director del Institute For Healthcare Management de ESADE. Profesordel Departamento de Dirección de Personas y Organización de ESADE. DoctorenAdministración de Empresas (ESADE-URL), España.

ISSN 1982-8829 Tempus, actas de saúde colet, Brasília, 13(2), 161-176, jun, 2019. 
ABSTRACT: Health systems have clearly contributed to improving the health status of the population, but the growth rate in health spending, higher than that of GDP growth in developed countries, raises questions about its sustainability. However, the analysis of sustainability tends to be partial: it focuses on health spending, and rarely on the coverage and tax revenues of the health system, a critical point in the case of Spain. In this article we analyze the impact of the economic crisis on the sustainability of the Spanish health system, and not only its economic impact, but also in terms of increasing health and inequalities. And finally, an estimate is made of the growth of public health expenditure in Spain for the next few years, in a scenario of reforms and in a growth scenario without structural changes, to highlight the cost of doing nothing. Changes and reforms become imperative, but this requires a climate of calm and open discussion that involves all the actors in the process, addrerrssing the real problems.

Keywords: Health Expenditure. Funding. Economic Crisis.

RESUMEN: Los sistemas sanitarios han contribuido de forma clara a la mejora del estado de salud de la población, pero el ritmo de crecimiento del gasto sanitario, superior al del crecimiento del PIB en los países desarrollados, presenta dudas sobre su sostenibilidad. Sin embargo, el análisis de la sostenibilidad tiende a ser parcial: se focaliza sobre el gasto sanitario, y pocas veces sobre las coberturas y los ingresos fiscales del sistema sanitario, punto crítico en el caso de España. En este artículo se analizan el impacto de la crisis económica en la sostenibilidad el sistema sanitario español, y no solo su impacto económico, sino en términos de salud y desigualdades crecientes. Y finalmente se hace una estimación del crecimiento del gasto sanitario público en España para los próximos años, en un escenario de reformas y en un escenario de crecimiento sin cambios estructurales, para poner en evidencia el coste de no hacer nada. Los cambios y reformas se hacen imperativos, pero esto requiere un clima de discusión sosegado y abierto que involucre a todos los actores del proceso, abordando los problemas reales.

Palabrasclave : Gasto Sanitario. Financiación. Crisis económica.

\section{INTRODUCCIÓN}

Este articulo analizaelescenario de sostenibilidad del sistema sanitario público de España para los próximos años, a partir de la evolución del gasto sanitario antes y durante el periodo de crisis económica del 2009 al 2015. Y se plantean dos cuestiones de fondo. En primer lugar, se pone sobre la mesa la parcialidad del debate sobre la sosteniblidad: a menudo el foco del debate se centra en el gasto sanitario y su crecimiento por encima del PIB, pero raramente se aborda la cuestión sobre el nivel de coberturas del sistema sanitario público ni la recaudación fiscal. En el fondo, la sostenibilidadeconómica depende de la capacidad de generar recursos y con ellos sufragar los gastos sanitarios incurridos para una población con un nivel determinado de coberturas. En segundo lugar, se pone de manifiesto la necesidad de poner en valor el resultado en salud del sistema, peor también su sostenibilidad social pues existen signos de desigualdades en salud crecientes tras la crisis económica. Para plantear el problema de la sostenibilidad, es necesario ir más allá de la 
óptica financiera: los cambios y reformas se hacen imperativos, pero esto requiere un clima de discusión sosegado y abierto que involucre a todos losactoresdelproceso, abordando los problemas reales.

\section{LA CONTRIBUCIÓN DE LOS SISTEMAS SANITARIOS A LA MEJORA DEL ESTADO DE SALUD}

La universalización de los sistemas sanitarios ha aportado una mejora significativa del estado de salud y ha contribuido a reducir las desigualdades sociales en los países desarrollados. La estructuración de los sistemas de salud de alcance universal ha sido uno de los avances sociales más significativos y más apreciados por los ciudadanos de los países de nuestro entorno social, cultural y económico. También es un rasgo común a todos ellos la gran confianza y buena valoración de la población en relación con su sistema de salud, sus profesionales y sus instituciones sanitarias.

La mejora significativa del estado de salud en general, y de la esperanza de vida en particular, es uno de los grandes avances de nuestra época, con una contribución importante (pero no exclusiva) de los sistemas sanitarios. Esta mejora se podría resumir en un dato impactante: durante el siglo XX la esperanza de vida en los países desarrollados se incrementó 3 meses por año (o 2,5 años por década). Este fenómeno se atribuye a múltiples factores, entre los que destacan la disminución de la mortalidad en las edades medias de la vida que se produjo en la primera mitad del siglo y la disminución de la mortalidad en las edades más avanzadas que ha predominado desde la década de 1970.

En España, en las últimas décadas han ido disminuyendo las tasas de mortalidad general y prematuray otros indicadores de efectividad como la esperanza de vida han aumentado de manera constante. Todas las comunidades autónomas han incrementado los indicadores de productividad de los servicios, lo que permite afirmar que la actividad de los servicios sanitarios está teniendo un efecto favorable para la salud.

Esta mejora del estado de salud se ha producido en todos los niveles socioeconómicos de la población. Puesto que el estado de salud se distribuye de forma desigual entre la población, con peores indicadores de salud entre los niveles socioeconómicos más desfavorecidos, se puede afirmar claramente que la mejora en la accesibilidad a los servicios sanitarios y su universalización han tenido y tienen un papel determinante en términos redistributivos.

\section{LA SOSTENIBILIDAD ECONÓMICA DEL SISTEMA SANITARIO ESPAÑOL}

\section{Un debate abordado de forma parcial}

Este éxito, sin embargo, ha tenido un coste significativo que preocupa a todos los gobiernos y a los responsables de los sistemas de salud. En las últimas décadas estamos asistiendo a un debate de fondo sobre la sostenibilidad económica de los sistemas sanitarios. Desde 1960, en los países 
de la OCDE el gasto sanitario ha crecido por encima delPIB. En los últimos quince años, dichos países han tenido un crecimiento anual del PIB de un 2,6\%, mientras que en el mismo periodo el crecimiento del gasto sanitario ha alcanzado un 3,9\% anual.En España, el gasto sanitario creció a una media anual del 8\% entre 1995 y 2008año en que estalla la crisis. Estos datos y su proyección futura están en la base de la preocupación por la capacidad de sostener financieramente el sistema sanitario público a largo plazo.

La evidencia muestra que el gasto en salud crece a medida que los países se desarrollan, si bien a un ritmo mayor que el crecimiento de su PIB (de media, 2 puntos por encima del PIB en los países de la OCDE). En consecuencia, el porcentaje del PIB destinado a sufragar el gasto sanitario se incrementa año tras año, lo que provoca el debate sobre la sostenibilidad financiera a medio y largo plazo de los sistemas de salud.

Figura 1. Evolución del porcentaje del PIB destinado a la salud en los países de la OCDE. 19602005

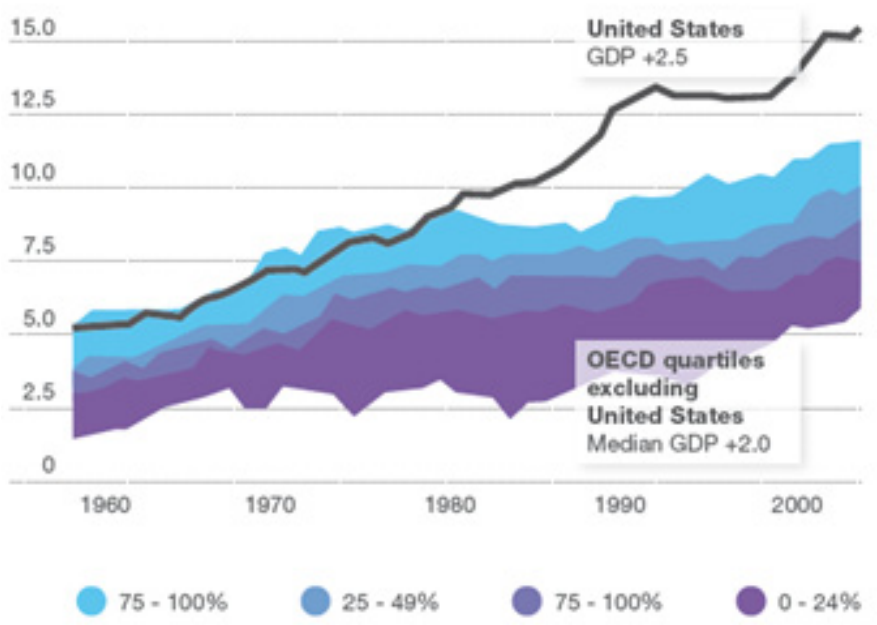

Fuente: Drouin, J.P., Hediger, V. y Henke, N. "Health care costs: A market-based view". The McKinsey Quarterly. Septiembre de 2008.

El debate macroeconómico sobre la sostenibilidad del sistema sanitario plantea la cuestión de cuánto gasto público se puede (criterio de disponibilidad de recursos) o debe (criterios de necesidad) asignar al sistema sanitario dentro del conjunto del gasto público. Dado que el criterio de necesidad supera al criterio de disponibilidad de recursos, el debate sobre la sostenibilidad está servido. Y este debate se ha acentuado en un contexto de déficit público y elevado endeudamiento.

No obstante, estedebate tiende a ser relativamente parcial, pues se centra exclusivamente en el gasto sanitario y obvia otras cuestiones también fundamentales: las coberturas del sistema sanitario (que necesidades queremos cubrir) o la política fiscal del país (cuantos recursos se pueden/deben recaudar). Y tampoco podemos obviar que el debate de la sostenibilidad del sistema no es una cuestión exclusivamente macroeconómica, sino que tiene que ir ligada a los resultados de salud que se hayan alcanzado. ¿Cuál es el coste de oportunidad de una sociedad con bajos niveles de 
salud? A pesar del problema evidente en cuanto a la futura sostenibilidad del sistema, la realidad es que este, hasta ahora, ha arrojado resultados positivos en cuanto a su objetivo principal, la mejora de la salud. Y los indicadores sobre estado de salud de la población española así lo indican.

\section{La cuestión fiscal: un factor clave en la sostenibilidad en España.}

La financiacióndel sistema sanitario proviene de los impuestos recaudados.Si se analiza la recaudación fiscal en España, se observa que, en comparación con nuestros socios europeos, cada año dejamos de recaudar entre 40.000 y 90.000 millones de euros. Si se compara la presión fiscal (porcentaje de impuestos recaudados sobre el PIB) en España (37,1\%) con la media de la Unión Europea (46,2\%), se detecta una diferencia de nueve puntos del PIB. Es decir, el sistema fiscal español es mucho menos eficiente que la media de los países europeos, pues, pese a que los tipos máximos de IVA, IRPF e Impuesto de Sociedades están entre los más elevados y por encima de la media europea, la recaudación obtenida es un 9,1\% del PIB inferior a la media europea. Considerando que, en grandes líneas, el PIB español es de un billón de euros, este 9\% de "no recaudación" ascendería a 90.000 millones y compensaría el déficit público de España, que en 2013 superó los 70.000 millones de euros para el conjunto de las administraciones públicas.

Figura2. Tipos fiscales y recaudación. Zona euro 2014.

\begin{tabular}{|l|ll|ll|ll|ll|}
\hline$\#$ & IRPF & & IVA & & Sociedades & & Ingresos (en \% & del \\
\hline 1 & Bélgica & 53,7 & Finlandia & 24 & Francia & 31,6 & Finlandia & 54,4 \\
\hline 2 & Portugal & 53 & Grecia & 23 & Malta & 35 & Francia & 51,8 \\
\hline 3 & España & 52 & Irlanda & 23 & Bélgica & 34 & Bélgica & 51 \\
\hline 4 & Países Bajos & 52 & Portugal & 23 & Portugal & 31,5 & Austria & 49,2 \\
\hline 5 & Finlandia & 51,1 & Italia & 22 & Italia & 31,4 & Italia & 47,7 \\
\hline 6 & Francia & 50,2 & Bélgica & 21 & España & 30 & Países Bajos & 46,4 \\
\hline 7 & Austria & 50 & España & 21 & Alemania & 29,8 & Media zona & 46,2 \\
\hline 8 & Eslovenia & 50 & Letonia & 21 & Luxemburgo & 29,2 & Alemania & 44,8 \\
\hline 9 & Alemania & 47,5 & Países Bajos & 21 & Grecia & 26 & Grecia & 44,6 \\
\hline 10 & Italia & 47,3 & Media zona & 20,5 & Media zona & 25,3 & Eslovenia & 44,2 \\
\hline 11 & Grecia & 46 & Austria & 20 & Austria & 25 & Luxemburgo & 43,7 \\
\hline 12 & Luxemburgo & 43,6 & Eslovaquia & 20 & Países Bajos & 25 & Portugal & 40,9 \\
\hline 13 & Media zona & 43,4 & Eslovenia & 20 & Finlandia & 24,5 & Malta & 40 \\
\hline 14 & Irlanda & 41 & Estonia & 20 & Eslovaquia & 23 & Estonia & 39,2 \\
\hline 15 & Chipre & 38,5 & Francia & 19,6 & Estonia & 21 & Chipre & 39 \\
\hline 16 & Malta & 35 & Alemania & 19 & Eslovenia & 17 & España & 37,1 \\
\hline 17 & Eslovaquia & 25 & Chipre & 18 & Letonia & 15 & Letonia & 35,1 \\
\hline 18 & Letonia & 24 & Malta & 18 & Irlanda & 12,5 & Irlanda & 34,5 \\
\hline 19 & Estonia & 21 & Luxemburgo & 15 & Chipre & 10 & Eslovaquia & 33,2 \\
\hline
\end{tabular}

Fuente: Eurostat Statistical Books. Taxation trends in the European Union. Data for the EU Member States, Iceland and Norway.2015 Edition.

ISSN 1982-8829 Tempus, actas de saúde colet, Brasília, 13(2), 161-176, jun, 2019. 
En conclusión, la cuestión sobre la sostenibilidad del sistema sanitario requiere una visión más global que abarque cuatro áreas:

1. En primer lugar, una reflexión colectiva sobre las prioridades del gasto público y el nivel de riqueza común (PIB) que la sociedad está dispuesta a dedicar a la salud.

2. En segundo lugar, una política fiscal eficaz que permita recaudar los recursos necesarios para financiar el conjunto del gasto público (y en particular el gasto sanitario). En este punto, existe un verdadero problema de insostenibilidad fiscal en España.

3. En tercer lugar, es necesario ahondar en el debate sobre coberturas del sistema (centrándose en el coste-efectividad de las prestaciones incluidasenlacartera de servicios más que enlapoblacióncubierta, laamplitud de lacarterahacia prestaciones no cubiertas como ondotologia o mal cubierta como cuidados sociosanitariosposthospitalariso)

4. Y, finalmente, y sin que ello signifique que sea menos importante, continuar con la única política que se ha impuesto hasta el momento: mejorar la gestión del coste de los procesos de asistencia sanitaria.

\section{EL IMPACTO DE LA CRISIS EN EL SISTEMA SANITARIO DE ESPAÑA}

Este debate sobre la sostenibilidad del sistema sanitario se ha visto indudablemente agravado por la reciente crisis económica sufrida en los países desarrollados, que ha introducido dos elementos importantes a tener en cuenta en el caso de España:

1. Un elevado déficit y deuda públicos que han obligado a reducir el gasto sanitario. Como esta reducción no se ha llevado a cabo de forma adecuada y las causas profundas del incremento del gasto no se han abordado ni reformado, los elementos sobre los que se ha actuado volverán a presionar para recuperarse rápidamente.

2. Un empobrecimiento de la sociedad, que incrementa el riesgo de desigualdades en salud. Como se ha demostrado en diversos estudios, las situaciones de pobreza generan condiciones de peor educación, alimentación y conocimiento y acceso al sistema, y menor capacidad de seguir los consejos de los profesionales sanitarios.

\section{LA EVOLUCIÓN DEL DÉFICIT PÚBLICO}

Una de las consecuencias negativas de la crisis que comenzó en 2008 ha sido el déficit público provocado por la caída de la recaudación fiscal y el crecimiento del gasto en protección social. Como destaca el siguiente gráfico, desde 2008 hasta la fecha se ha generado déficit durante todos los años, si bien es cierto que en los últimos se puede apreciar una reducción de éste. 
Figura 3.EjecucióndelPresupuestodel Estado. Ingresos y gastos (en millones). España, 20062017.

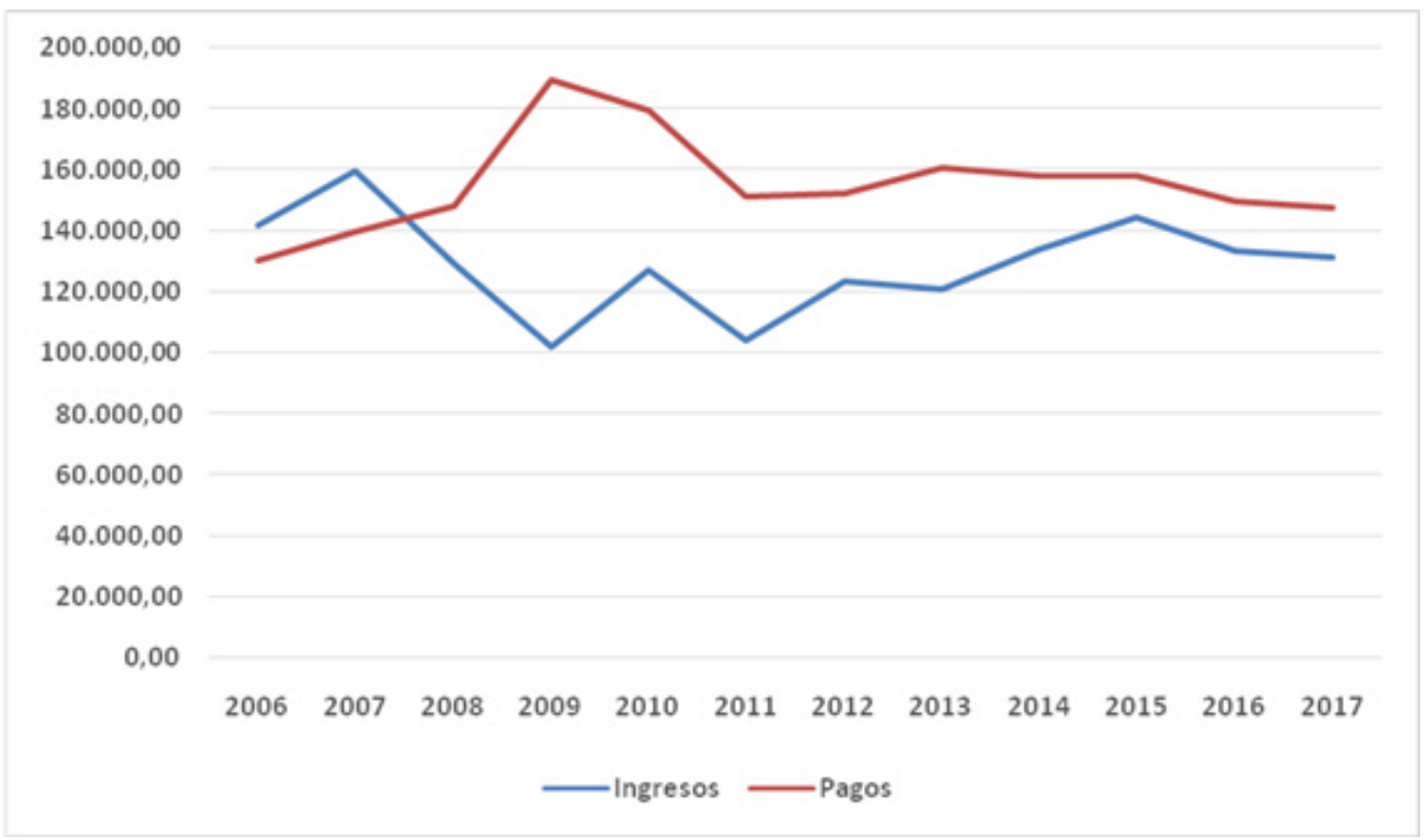

Fuente: Síntesis de Indicadores Económicos. Tablas completas. Ministerio de Economía, Industria y Competitividad (2018)

No obstante, cabe destacar que la contribución a la reducción del déficit ha sido asimétrica entre las diferentes administraciones. Si se analiza el gasto público en operaciones no financieras entre 2010 y 2015 (años de mayor esfuerzo de consolidación fiscal), se observa que el gasto corriente se ha reducido significativamente en las comunidades autónomas y en los ayuntamientos (-15\% y $-9 \%$ respectivamente), mientras que se ha incrementado en la Seguridad Social y la Administración General del Estado no ha contribuido a reducir el gasto público.

Figura 4. El gasto enlasadministraciones públicas enoperacionesfinancieras, España 2010-2015.

\begin{tabular}{|l|r|r|r|r|}
\hline & 2010 & 2.015 & Var. 2010-2015 & $\%$ Var. \\
\hline Estado & 222.649 & 221.364 & -1.285 & $-0,6 \%$ \\
\hline Seguridad Social & 157.606 & 159.544 & 1.938 & $1,2 \%$ \\
\hline Comunidades autónomas & 188.118 & 170.976 & -17.142 & $-9,1 \%$ \\
\hline Entes locales & 76.613 & 64.699 & -11.914 & $-15,6 \%$ \\
\hline Subtotal gasto público & 644.986 & 616.583 & -28.403 & $-4,4 \%$ \\
\hline Transferencias entre AAPP & 151.880 & 145.881 & -5.999 & $-3,9 \%$ \\
\hline Gasto público AAPP & 493.106 & 470.702 & -22.404 & $-4,5 \%$ \\
\hline Déficit AAPP & -102.217 & -54.628 & 47.589 & $-46,6 \%$ \\
\hline
\end{tabular}

Fuente: IGAE. Contabilidad nacional. Operaciones no financieras del sector Administraciones Públicas y sus subsectores. Serie histórica 1995-2015. SEC 2010.

ISSN 1982-8829 Tempus, actas de saúde colet, Brasília, 13(2), 161-176, jun, 2019. 


\section{La evolucióndel gasto sanitario durante lacrisis}

Desde que se iniciólacrisis económica en 2008, el gasto sanitario público fue disminuyendo y se situó en 2013 en 61.710 millones de euros, 8.900 millones menos que en 2009. Y a partir de 2015 el gasto sanitario se recupera

Figura 5. Estadística de gasto sanitario público (en millones). España, 1995-2016 (último año publicado)

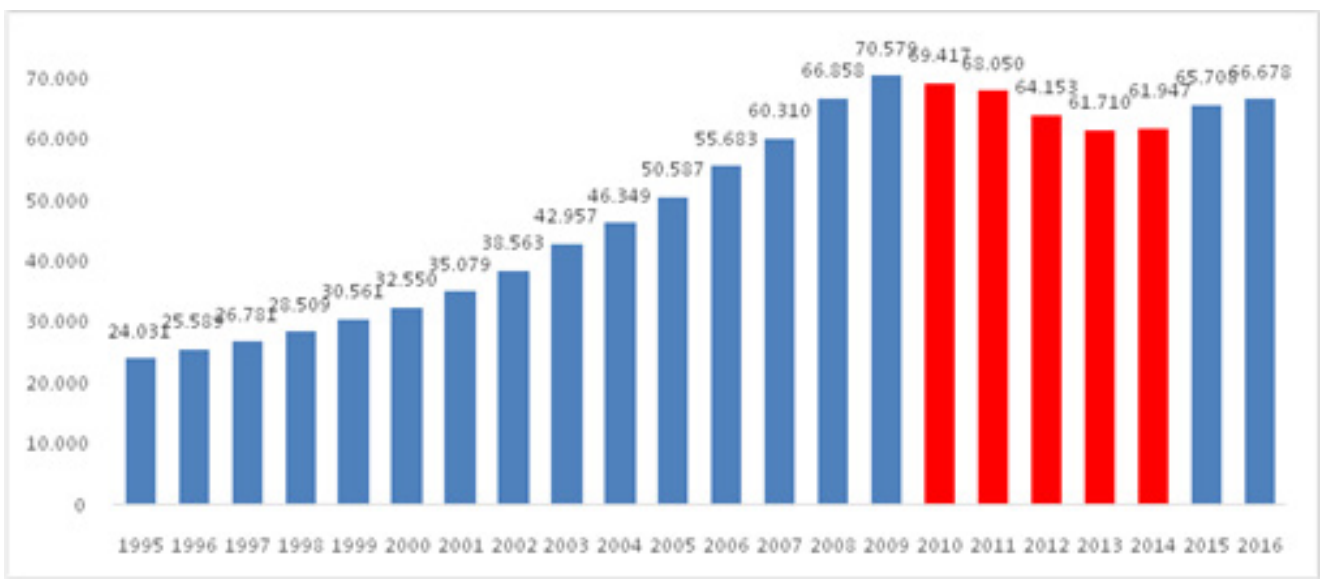

Fuente: Estadística de Gasto Sanitario Público 2017. Ministerio de Sanidad, Servicios Sociales e Igualdad.

La reduccióndel gasto sanitario ha sido significativa y evidente, pero es conveniente analizar cómo se ha reducido y a qué se atribuye dicha importante disminución.

- En términos relativos, destaca la disminución de los gastos de capital(las inversiones), con una reducción del 201\% (el gasto actual se eleva a 850 millones y la reducción ha sido de 1.707 millones).

- En términos relativos, cabe destacar también que la reducción es proporcionalmente mucho más importante en Servicios de Prevención y Salud Pública, y Servicios de Atención Primara, que en la Atención Hospitalaria.

- En términos absolutos, la mayor reducción se concentra en Remuneración del personal, con una disminución de casi 3.500 millones de euros

- A continuación, le sigue el gasto en Farmacia: 3.000 millones en valores absolutos

A la luz de los datos, los resultados indican que no ha habido ningún cambio sustancial en la naturaleza del gasto sanitario, lo que pone de relieve la rigidez del sistema sanitario frente a cambios estructurales. Estas rigideces pueden explicarse por el marco de relaciones laborales de carácter estatutario, laescasa (pornodecir nula) autonomía de gestión de los centros, la baja integracióndeentreatención primaria y atención especializada, una gestiónpresupuestaria que limita los vasos comunicantes entre partidas de gasto, etc. 
Las medidas a adoptar suelen ser impopulares y requieren de una alta comprensión del problema y de sus implicaciones. Por ello, los equipos de gobierno de las diferentes administraciones implicadas difícilmente plantean este tipo de medidas que muestran pocos beneficios tangibles a corto plazo.

\section{El impacto de la crisis en la salud y la desigualdad}

La relación entre crisis económica y salud ha sido analizada en varios estudios, sin clarificarse por completo. Efectivamente, este enfoque es complejo por problemas metodológicos (datos agregados en los resultados, desfase entre la crisis de hoy y los efectos a medio plazo) y por dificultades asociadas a la divergencia de los efectos de la crisis sobre la salud según la problemática. Se puede mencionar el ejemplo de una correlación positiva entre el desempleo y los trastornos mentales e incluso el suicidio o el consumo de alcohol, pero negativa entre el desempleo y los accidentes de tráfico.

En primer lugar, conviene distinguir tres posibles tipos de efectos de la crisis económica:

1) El impacto en los sistemas de salud

2) El impacto en el estado de salud.

3) El impacto en las desigualdades sociales en salud.

El impacto en los sistemas de salud ya se ha expuesto y es evidente: la reducción del gasto público en salud, una diminución de 8.900 millones de euros en España entre 2009 y 2014. Se observa en paralelo un aumento de las contribuciones individuales con respecto a los medicamentos en España. Además, en ocasiones se ha restringido a ciertos segmentos de la población su acceso a la atención sanitaria (en España los extranjeros en situación ilegal solo disponen de cobertura sanitaria de emergencias y de maternidad). Entre los otros impactos observados, destacan la disminución del precio de los medicamentos, la disminución del número de prescripciones y la limitación e incluso reducción del empleo público en el sector de la salud.

Por el contrario, es más difícil caracterizar el impacto directo de la crisis en el estado de salud de la población. La correlación entre gasto público en salud, mortalidad y esperanza de vida es muy baja a corto plazo. En los países más afectados por la crisis, algunos indicadores muestran que la crisis podría tener un impacto en ciertos problemas de salud o en poblaciones específicas. Los problemas de salud mental, por ejemplo, van en aumento (progresión de la prevalencia a un año en Grecia, aumento de las consultas psiquiátricas en España..., todo ello acompañado por una tendencia al alza en la tasa de suicidios). En cuanto a otros indicadores, las pruebas son muy limitadas y pueden estar relacionadas con factores coyunturales que explicarían las variaciones observadas en el estado de la salud. Por lo tanto, sería necesaria una búsqueda más profunda y más

ISSN 1982-8829 Tempus, actas de saúde colet, Brasília, 13(2), 161-176, jun, 2019. 
exhaustiva para estudiar el impacto de la crisis en el estado de la salud.

Finalmente, el impacto más evidente de la crisis económica es el relativo al incremento de las desigualdades sociales. Entre 2009 y 2012, el número de personas en riesgo de pobreza o de exclusión social aumentó en nueve millones en la Unión Europea; y según el análisis del índice Gini, que mide la diferencia de recursos económicos entre el $20 \%$ de la población más rica y el 20\% de la población más pobre de un Estado o región, las desigualdades dentro de la Unión Europea han aumentado. Un estudio particularmente interesante sobre esta cuestión fue publicado por la Agencia de Calidad y Evaluación Sanitarias de Cataluña. El estudio se centra en la población infantil en riesgo de pobreza, cuyo riesgo de exclusión social ha aumentado más rápidamente que el de otras categorías de edad. El estudio muestra que el estado de salud de esta población no ha evolucionado desfavorablemente, aunque sí que lo han hecho los indicadores de la calidad y modo de vida, que se han deteriorado: menos actividad física, malos hábitos alimentarios, mucha más obesidad...

Sobre este tercer grupo de impactos, el de las desigualdades sociales, las respuestas observadas en cuanto a las políticas públicas son más heterogéneas. En los países dotados de sistemas amplios de protección social, el aumento de las desigualdades sociales ha sido limitado (y en países como Alemania, los Países Bajos o Suecia el índice Gini incluso ha disminuido). Los países más afectados por la crisis (excepto Islandia) y que disponen de sistemas de protección social más frágiles serán aquellos donde probablemente las consecuencias de la crisis en términos de salud serán más importantes a medio plazo

\section{Desigualdad y sistema sanitario: un debate del que no se habla}

Además del posible impacto de lacrisisenlasalud de lapoblación y ladesigualdad, enEspaña, persistenciertas desigualdades enel sistema sanitarioque deberían abordarse.

1) La distribucióndel estado de salud entre lapoblación: La recientecrisis económica há traído de nuevo elevados niveles de desempleo y mayores niveles de pobreza sostenida, por lo que cabe esperar un efecto a medio y largo plazo sobre la salud. Aunque estas fuentes de desigualdad no se han generado en el sistema sanitario, este sí puede y debe contribuir a mitigarlas. Y la universalización del acceso, especialmente el acceso a la atención primaria y las urgencias, supone un vector importante para esa atenuación

2) El tiempo de acceso: Si bien es cierto que la atención primaria ha contribuido en gran medida a la mejora del acceso a los servicios sanitarios (con un efecto muy positivo en las clases más desfavorecidas), la presencia de listas de espera genera una fuente de desigualdad en la atención especializada. La oportunidad del doble aseguramiento público/privado del que pueden beneficiarse las rentas más favorecidas permite que dichos ciudadanos puedan evitar los tiempos de espera.

Tempus, actas de saúde colet, Brasília, 13(2), 161-176, jun, 2019. ISSN 1982-8829 
3) El efecto de loscopagos: Los copagos, cuandoexisten y son significativos, pueden tener el efecto de incrementar las desigualdades en salud. Pero se ha asociado con demasiada ligereza con los conceptos de universalización de la cobertura y acceso gratuito (ausencia de copago en los servicios sanitarios). En un contexto de escasez de recursos para financiar la sanidad, no abordar un modelo de copago para la asistencia (y no solo para medicamentos) con buen sistema de exenciones proteja a las clases desfavorecidas (el diseño mayoritario en países de nuestro entorno), significa también renunciar a que se generan recursos adicionales para reducir las desigualdades en salud (presentes en mayor medida en las rentas desfavorecidas).

4) La capacidad de navegación por el sistema o Health Literacy: A niveleuropeo (The European Health Literacy Project) se ha observado, a partir de una encuesta a personas adultas en ocho países (Alemania, Austria, Bulgaria y España, entre otros), que en cerca de la mitad de las personas encuestadas ( $47 \%$ de 8.000 ) las principales variables relacionadas con mejores niveles de comprensión eran: sexo femenino, menor edad, mayor nivel económico y educativo y mayor estatus social. En España la alfabetización en salud ha sido estudiada por la Universidad de los Pacientes, que identificó que el $62 \%$ de los encuestados afirmaba no tener el conocimiento suficiente para manejar suenfermedad, y que dichoconocimientomejorabaconelnivel de ingresos.

5) La financiación autonómica y su impacto enel gasto sanitário: El gasto sanitario por habitante presenta diferencias sustanciales entre los diversos territorios, superando los 40 puntos porcentuales. Por ejemplo, el gasto en el País Vasco en 2016 fue un 42\% más elevado que enAndalucía.

6) Los subsistemas de gestión privada de la cobertura pública de funcionarios públicos:Enel subsistema de aseguramiento de funcionarios civiles, judiciales y militares, comúnmente conocido como sistema MUFACE (Administración Civil del Estado), MUGEJU (Administración de Justicia) e ISFAS (Fuerzas Armadas) los propios funcionarios del Estado eligen libre y voluntariamente si quieren ser atendidos por el Sistema Nacional de Salud (SNS) o por una aseguradora. Este es, sin duda, un hecho que genera desigualdad, ya que es una opción que no tienenel resto de ciudadanos que cotizanvíalaseguridad social.

\section{LA PROYECCIÓN DEL GASTO SANITARIO A LARGO PLAZO EN ESPAÑA}

Finalmente, para completar el debate sobre la sostenibilidad del sistema sanitario debemos abordar la proyección del gasto sanitario a largo plazo. Para ello, se han utilizadodos enfoques basados en los modelos de proyección anteriormente citados. Estos dos enfoques permiten situar un rango plausible de evolución del gasto sanitario público en España para los próximos diez años:

El primer enfoque basa la proyección del gasto en la tasa de crecimiento histórica del gasto sanitario. Este escenario parte de la hipótesis subyacente en el informe del World Economic Forum, que prevé un escenario continuista en el que no habrá reformas estructurales de fondo que permitan modificar el crecimiento del gasto sanitario. Según estos modelos de proyección (que son los más

ISSN 1982-8829 Tempus, actas de saúde colet, Brasília, 13(2), 161-176, jun, 2019. 
habituales), el gasto sanitario sobre el PIB podría superar el 20\% en algunos países en el año 2040 si no se producen cambios fundamentales en las causas del gasto.

Este escenario se ha construido a partir de la proyección de la tasa de crecimiento del gasto sanitario, que en la serie histórica analizada (1995-2012) fue del 6,1\% anual. Dentro de esta serie histórica, el gasto sanitario público creció al 8,2\% entre 1995 y 2009 (antes del ajuste del gasto público) y decreció a una tasa del 3,1\% anual entre 2010 y 2012. Esta tasa se ha corregido ligeramente en los primeros años de la proyección, puesto que en los últimos años el crecimiento ha sido negativo. En este sentido, se ha hecho una previsión de crecimiento moderado del gasto hasta alcanzar una tasa constante del $6,1 \%$.

El segundo modelo de proyección se basa en los estudios realizados por la UE, que proyectan el gasto sanitario público en porcentaje sobre el PIB bajo la premisa de que el crecimiento no será lineal, tal como se ha señalado en capítulos anteriores. Las principales hipótesis que baraja este modelo son:

- La mitad de los futuros años de vida ganados gracias al aumento de la esperanza de vida se obtendrán en buenas condiciones de salud.

- Se prevé que los Estados puedan introducir medidas activas de contención de gastos.

- Se prevé que ciertos costes no crezcan por igual en los países desarrollados: la innovación tecnológica (los escenarios actuales subestiman su impacto en la contención de los costes), la productividad de la economía (que se prevé que crezca a un menor ritmo en el futuro, lo que afectará positivamente a la diferencia que había con respecto a la del sector salud), los salarios (que en determinados países se contendrán en mayor medida de lo que lo han hecho hasta la fecha) y los productos sanitarios y la infraestructura (dependiendo del país, su evolución podrá ser gestionada por los Estados miembros ).

En el fondo, el modelo de la UE parte de la premisa de que los Estados miembros introducirán mecanismos y/o reformas que permitirán gestionar la tasa de crecimiento histórica del gasto.

En el caso de España, la UE prevé un incremento del gasto sanitario público de entre 1,1 y 1,9 puntos sobre el PIB para el conjunto periodo 2013-2060, situándolo entre el 7\% y el 7,8\%. En la proyección realizada se han considerado dos hipótesis:

- El porcentaje de gasto sanitario se incrementará anualmente en 0,1 puntos porcentuales.

- $\quad$ El PIB crecerá a una tasa del 2,7\% y el 2,3\% en 2016 y 2017 (Fuente: FMI), y del 2,5\% hasta 2025 (Fuente: Crecimiento PIB 2018-2025: La economía española en 2033, PWC). 
Los resultados de ambos modelos muestran un rango de incremento del gasto sobre 2015 de entre 32.000 y 48.000 millones. Considerando que el gasto sanitario público en 2015 se habrá situado previsiblemente en 64.833 millones de euros, esto significa que para 2025 se podría estimar entre 97.000 y 113.000 millones anuales.

Figura6. Análisis comparado de los dos escenarios de necesidades de financiación

\begin{tabular}{|lrrr|}
\hline \multicolumn{1}{|c}{ Año } & 2015 & 2025 (Esc. 1) & 2025 (Esc. 2) \\
\hline Gasto actual & 64.833 (E) & 10 & 10 \\
\hline Años & $5,8 \%$ & $4,2 \%$ \\
Tasa crecimiento gasto público & 113.694 & 97.406 \\
Gasto público previsto & 48.862 & 32.573 \\
Incremento absoluto sobre 2015 & 236.027 & 172.251 \\
Necesidades financieras & & & \\
\hline
\end{tabular}

\section{Cómose puede interpretar este rango?}

Si la diferencia entre ambos modelos se basa en una estimación lineal o una estimación "gestionada" del gasto sanitario público, los 16.00 millones de diferencia entre ambos modelos representan el coste de "no hacer nada", es decir, las necesidades de financiación del gasto público sanitario atribuibles a no tomar medidas suficientes para gestionar el crecimiento del gasto sanitario. Pero también significa que los 32.500millones de euros estimados en el escenario posibilista representan la base que habrá que financiar para cubrir las necesidades del sistema sanitario público. ¿El crecimientodel PIB y/o lasmejorasenlarecaudación fiscal permitirán financiar estas necesidades?

Figura7. Las necesidades de financiación por tramos

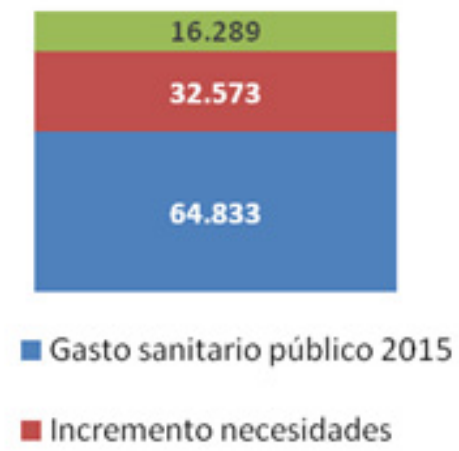

\section{Podremos afrontar la factura?}

Si solo lo miramos desde la óptica financiera, se está imponiendo la visión de que no podremos asumirla. Sin embargo, quizá deberíamos analizar esta cuestión desde una perspectiva más amplia para poder contestar con mayor criterio a esta pregunta.

ISSN 1982-8829 Tempus, actas de saúde colet, Brasília, 13(2), 161-176, jun, 2019. 
¿Sabemos lo que hay que hacer para afrontar este escenario?

Parece que sí. En los últimos años se han multiplicado los informes y estudios publicados sobre los cambios y/o reformas a introducir en el sistema sanitario. Y entre ellos existe un mínimo común denominador de medidas a adoptar que supone una sólida base para iniciar dichoscambios.

Entonces, ¿por qué no se hace?

Afrontar este escenariorequiereun clima de discusión sosegado y abierto que permita entrar a fondo en aspectos ciertamente complejos y que no permiten respuestas sencillas. Y esto requiere una gran responsabilidad de todos los actores involucrados (profesionales sanitarios, gestores, políticos, agentes sociales y sociedad) para analizar la situación y transmitir los mensajes de forma clara, aunque sin caer en desatinadas simplificaciones, a menudo más ideológicas que técnicas, que acaban por no abordar los problemas reales y por no aportar las soluciones necesarias.

Una última reflexión: el coste de no hacer nada es invisible (nunca tendremos dos gastos sanitarios que comparar) y es más importante en el futuro que en el corto plazo. En este sentido, ¿qué incentivos hay para actuar ahora desde el ámbito de las políticas públicas?

Figura 8. Evolucióndel gasto sanitarioestimado segúnlos dos escenarios previstos.

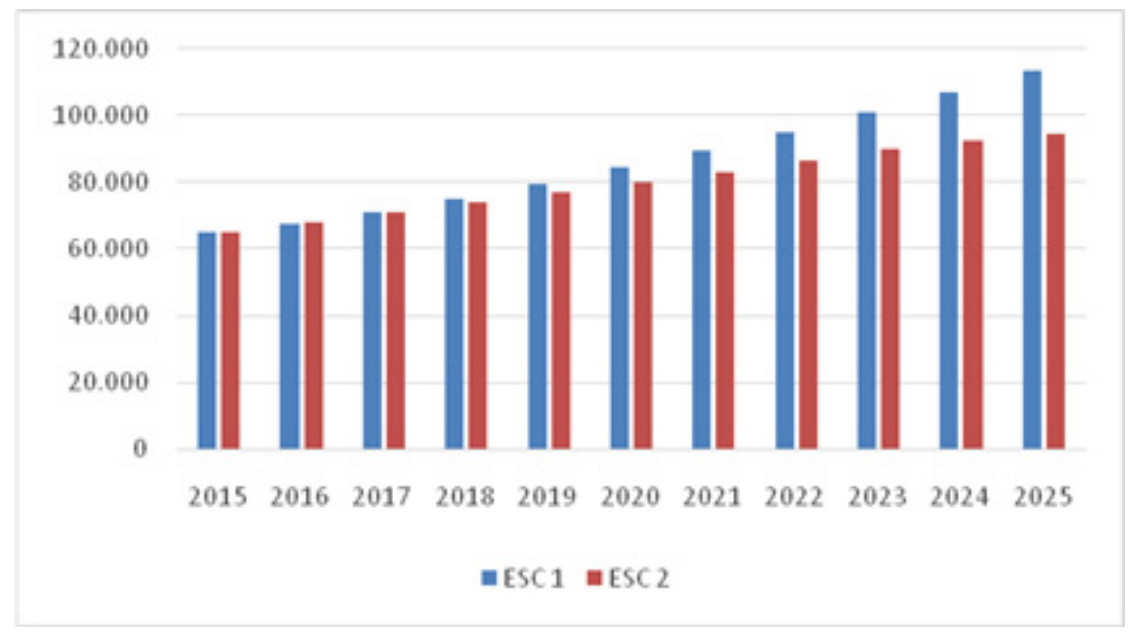

\section{Contribuição dos autores:}

Os autores participaram da redação completa do manuscrito, considerando a hipótese de trabalho, a busca de dados secundários, o desenho do modelo de projeção do gasto em saúde e a escrita.

\section{REFERÊNCIAS}

1. Agència de Qualitat i Avaluació Sanitàries de Catalunya (AQuAS). Central de Resultats. "Efectes de la crisi econòmica en la població infantil de Catalunya". Barcelona (ESP): AQuAS. 2014. 10 páginas. 
2. De La Maisonneuve, C. y Oliveira Martins, J. "A projection method for public health and long-term care expenditures". Economics Department Working Papers núm. 1048, OCDE, París. 2013.

3. Dever GEA. An Epidemiology Model for Health Analysis. Soc Ind Res 1976; vol. 2, pág. 465.

4. DomínguezBarrero, F., López Laborda, J. y Rodríguez Sauco, F. "El hueco que deja el diablo: una estimación del fraude en el IRPF con microdatos tributarios”. Estudios sobre la Economía Española. FEDEA. 2014.

5. Drouin, J.P., Hediger, V. y Henke, N. "Health carecosts: A market-basedview". The McKinsey Quarterly. Septiembre de 2008.

6. Economist Intelligence Unit. "The future of healthcare in Europe. A report from the Economist Intelligence Unit sponsored by Janssen”. Londres: The Economist Intelligence Unit Limited. 2011.

7. European Observatory on Health Systems Policies. "Health, health systems and economic crisis in Europe". En: Impact and policy implications. Bruselas: Organización Mundial de la Salud. 2013.4.

8. Eurostat Statistical Books. Taxation trends in the European Union. Data for the EU Member States, Iceland and Norway. 2015 Edition.

9. Gispert, R., Torné, M. y Arán, M. “La efectividad del sistema sanitario en España”. Gaceta Sanitaria. 2006; 20 (supl. 1): 117-26.

10. Gómez Serrano, P.J. y Molero, R. "El incremento de la desigualdad y la exclusión social en la UE15 y sus determinantes". Fundación Foessa. 2014.

11. Karanikolos, M., Mladovsky, P., Cylus, J., Thomson, S., Basu, S., Stuckler, D. et al. "Financial crisis, austerity, and health in Europe". The Lancet, 2013; 381(9874): 1323-31.

12. McGovern, L., Miller, G. y Hughes-Cromwick, P. "The Relative Contribution of Multiple Determinants to Health Outcomes”. Health Policy Brief (Health Affairs). 21 de agosto, 2014.

13. Mestres, J., Suárez-Lledó, J., Canals, C., Gutiérrez-Domènech, M. Dossier: “El retodemográfico". En: Hernández, A., Aspachs, O., Fernández, E. y Gual, J. IM10. Barcelona: CaixaBank Research. 2015. pp. 32-38.

14. Mohr, P.E., Mueller, C., Neumann, P., Franco, S., Milet, M., Silver, L. et al. "The Impact of Medical Technology on Future Health Care Cost. Final Report”. Project Hope. Bethesda,

ISSN 1982-8829 Tempus, actas de saúde colet, Brasília, 13(2), 161-176, jun, 2019. 
Maryland: Center for Health Affairs. 2011.

15. Navarro, R. (dir.). “Análisis de la sanidad en España a lo largo del siglo XX”. Madrid: Instituto de Salud Carlos III, Ministerio de Sanidad y Consumo. 2002.

16. OECD Health Policy Studies. "Value for Money in Health Spending”. París: OECD Publishing. 2010.

17. Robles, E., García F. y Bernabeu, J. "La transición sanitaria en España desde 1900 a 1990”. Revista Española de Salud Pública. 1996; 70: 221-223.

18. Segura Benedicto, A. "Recortes, austeridad y salud". Informe SESPAS 2014. Gaceta Sanitaria. 2014; 28(S1): 7-11.

19. Simonato, L., Ballard, T., Bellini, P. y Winkelmann, R. "Avoidable mortality in Europe 1955-1994: a plea for prevention". Journal of Epidemiology and Community Health. 1998, $52(10)$

20. Smith, S., Newhouse, J.P. y Freeland, M.S. "Income, Insurance, and Technology: Why Does Health Spending Outpace Economic Growth?". Health Affairs. 2009; 28: 1276-84.

21. Vaupel, J.W. "Understanding longevity and how to improve it". En: Global Forum for Longevity. París: Axa. 2011. pp. 44-51.

22. Westerling, R. "Decreasing gender differences in 'avoidable' mortality in Sweden". Scandinavian Journal of Public Health. 2003, 31(5): 342-9.

23. World Economic Forum. "Sustainable Health Systems: Visions, Strategies, Critical Uncertainties and Scenarios". 2013.

Artigo apresentado em: março de 2019. Artigo aprovado em: abril de 2019 Conflito de interesses: os autores declaram não haver conflitos de interesses Suporte financeiro: não houve financiamento 